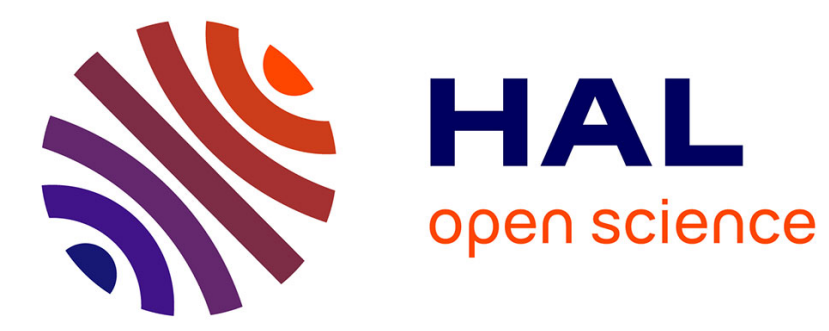

\title{
Evolution des indices catégoriels en purépecha
}

Claudine Chamoreau

\section{To cite this version:}

Claudine Chamoreau. Evolution des indices catégoriels en purépecha. Faits de langues, 1999, 14, pp.143-152. halshs-00293825v2

\section{HAL Id: halshs-00293825 \\ https://shs.hal.science/halshs-00293825v2}

Submitted on 10 Jul 2008

HAL is a multi-disciplinary open access archive for the deposit and dissemination of scientific research documents, whether they are published or not. The documents may come from teaching and research institutions in France or abroad, or from public or private research centers.
L'archive ouverte pluridisciplinaire HAL, est destinée au dépôt et à la diffusion de documents scientifiques de niveau recherche, publiés ou non, émanant des établissements d'enseignement et de recherche français ou étrangers, des laboratoires publics ou privés. 


\section{Evolution des indices catégoriels en purépecha}

Claudine CHAMOREAU*

Le purépecha (connu aussi comme tarasque) est parlé au centre-ouest du Mexique. Dans les premières descriptions du XVIème siècle, Maturino Gilberti et Juan Bautista de Lagunas ${ }^{1}$ présentent des unités qui accompagnent les noms. Ces élèments apportent des informations sur l'objet qui est représenté par le nom : les caractéristiques physiques (rond, long, plat) ou la façon dont l'objet ou les objets sont présentés (type de disposition, volume occupé, etc...).

Aujourd'hui, ces unités (celles qui existent encore) sont liées à la quantification d'objets. Pour compter une ou plusieurs entités, on utilise un numéral auquel peut être postposée une de ces unités.

L'objectif de cette étude est de montrer l'évolution de ces éléments. Du XVIème siècle à nos jours, le nombre de ces unités a considérablement diminué. Ce facteur quantitatif est important mais il est surtout révélateur d'une réorganisation sémantique.

\section{DES PRECISIONS TERMINOLOGIQUES}

Dans le cadre de la théorie fonctionnaliste, toute langue est constituée de classes dont l'inventaire s'établit en utilisant des critères syntaxiques. Appartiennent à une classe, les unités qui présentent les mêmes compatibilités et qui s'excluent mutuellement en un point de l'énoncé. Le premier critère se situe sur l'axe syntagmatique et le deuxième sur l'axe paradigmatique. Dans la langue purépecha, différentes classes ont ainsi été dégagées, parmi lesquelles se trouvent la classe des noms et la classe des numéraux. Les unités qui appartiennent à la classe des noms ne présentent pas d'opposition de genre. En revanche, il existe des unités qui permettent de ranger des noms en différentes catégories. Ces procédés de catégorisation apparaissent lorsque l'objet ou les objets qui sont représentés par le ou les noms sont comptés. Dans cette optique, la désignation qui est généralement utilisée (classificateur numéral) comporte une certaine ambigüité, c'est pourquoi, je préfère nommer les unités indices catégoriels de la classe nominale.

\section{LA DYNAMIQUE DES INDICES CATEGORIELS AU XVIEME SIECLE}

Le franciscain Maturino Gilberti a écrit en 1558 la première grammaire décrivant cette langue. Cette œuvre remarquable présente un inventaire de ce que l'auteur appela "les particules pour les choses".

\footnotetext{
* THEDEL, Université René Descartes, Paris V et CELIA, UMR 7595 du CNRS.

1 Gilberti, 1987 [1558], p. 275 et Lagunas, 1983 [1574], p. 168-169.
} 


\begin{tabular}{|c|c|}
\hline $\begin{array}{c}\text { Indices } \\
\text { catégoriels }\end{array}$ & Sens \\
\hline yra & pour les choses rondes (comme des oranges) \\
\hline quira & idem \\
\hline$y c h a$ & pour les choses longues (comme des bâtons ou des cierges) \\
\hline echu & pour les choses larges et plates (comme du papier ou des tortillas) \\
\hline chere & pour les choses tissées (comme des draps) \\
\hline thumbi & $\begin{array}{l}\text { pour les choses comme des sacs, des sacoches, des charges ou des régimes de } \\
\text { bananes }\end{array}$ \\
\hline çutu & idem sauf pour les régimes de bananes \\
\hline xancha & pour les choses à quatre pieds (comme les animaux ou les bancs) \\
\hline chuui & idem et aussi pour les poules et la volaille \\
\hline hutu & pour les choses comme les bottes ou les fagots \\
\hline anga & pour mettre une chose debout ou une chose dressée \\
\hline umba & $\begin{array}{l}\text { pour les choses entassées (comme les oranges, les personnes et tout autre } \\
\text { chose) }\end{array}$ \\
\hline tzuhti & pour deux choses entassées \\
\hline thsinguui & $\begin{array}{l}\text { pour les choses comme les plats, les écuelles, les tasses lorsqu'ils sont mis les } \\
\text { uns sur les autres }\end{array}$ \\
\hline para & pour les tasses (faites en écorce) lorsqu'elles sont séparées \\
\hline tacu & $\begin{array}{l}\text { pour les choses pliées ou plates et larges comme les livres et les tapis en } \\
\text { grande quantité (plus de un) }\end{array}$ \\
\hline churu & pour les choses qui s'entassent sans ordre (comme les oranges) \\
\hline chungua & idem \\
\hline
\end{tabular}

La première colonne de ce tableau présente les formes telles qu'elles sont transcrites par Gilberti. La deuxième colonne indique le sens des indices tel qu'il est donné par Gilberti et précisé par Juan Bautista de Lagunas. Cet auteur a repris l'inventaire de Gilberti, en respectant l'ordre mais en précisant le sens de certains éléments.

Cet inventaire comporte dix-huit unités ${ }^{2}$. Les trois premiers éléments yra (si on ne tient pas compte de quira, qui semble être une variante), ycha et echu sont ceux qui sont encore utilisés aujourd'hui. Il semble donc que Gilberti a rangé ces unités par ordre de fréquence et de productivité. De plus, le sens de ces trois éléments n'est motivé que par une caractéristique physique de l'objet, respectivement [rond], [long] et [plat]. Il existe une certaine redondance entre les informations apportées par les indices et les particularités physiques des objets. Les traits sémantiques ([rond], [long] et [plat]) reprennent uniquement les propriétés définitoires et générales de l'objet référé par le nom.

\begin{tabular}{|l|l|l|}
\hline yra (quira) 3 & ycha & echu \\
\hline [rond] & {$[$ long] } & [plat] \\
\hline
\end{tabular}

Ces trois indices catégoriels sont fortement liés à la quantification des objets, puisque le locuteur ne s'attache pas à préciser leur disposition, leur volume, etc. Ces unités s'excluent mutuellement, par exemple, avec naransa "orange", c'est yra qui convient, avec siranta "papier", on utilise echu et chucari "bûche" est accompagné de ycha. Dans chaque

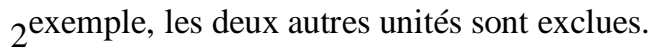

Parmi les autres indices catégoriels, deux sont aussi liés à des particularités physiques des objets. Cependant, on considère la quantité des éléments de soutien (pieds ou pattes) et non leur forme physique (pour les animaux, notamment) :

\begin{tabular}{|l|l|}
\hline xancha & chuui \\
\hline [quadripède] & [bipède] \\
\hline
\end{tabular}

\footnotetext{
2 On peut sinterroger comme le fait, de façon pertinente, Fernando Nava sur le nombre exact de ces unités et sur leur utilisation exacte au XVI ${ }^{\text {ème }}$ siècle.

${ }^{3}$ Entre parenthèses, se trouvent les unités désignées comme des variantes.
} 
Les autres indices catégoriels sont de nature à instaurer une catégorisation contextuelle, liée à l'activité et au fonctionnement humain. Il n'est pas fait uniquement référence aux propriétés physiques des objets mais aussi à leur mesure (le volume occupé par plusieurs objets), à leur disposition, à leur mode particulier d'entassement. Le locuteur indique donc la représentation spatiale de ces objets.

\begin{tabular}{|l|l|l|}
\hline thumbi (çutu) & hutu & chere \\
\hline [rond] & {$[$ long] } & [plat] \\
\hline \multicolumn{3}{|c|}{ [volume] } \\
\hline
\end{tabular}

\begin{tabular}{|l|l|l|}
\hline para & anga & tacu \\
\hline [rond] & {$[$ long] } & [plat] \\
\hline \multicolumn{3}{|c|}{ [disposition] } \\
\hline
\end{tabular}

\begin{tabular}{|l|l|l|}
\begin{tabular}{|l|l|} 
umba (pluriel) \\
tzuhti (duel) $)^{4}$
\end{tabular} & churu (chungua) & thsinguui \\
\hline [+ordonné] & [-ordonné] & [plat] \\
\hline \multicolumn{3}{|c}{} \\
[entassement]
\end{tabular}

Ces qualités sont temporaires, spécifiques et contextuelles. Par conséquent, un même objet, naransa "orange" peut être accompagné, par exemple, de yra si l'on veut insister sur l'objet en lui-même (uniquement compter le nombre d'oranges), de anga si on dispose plusieurs oranges les unes sur les autres, de umba si plusieurs oranges sont entassées de façon ordonnée, de tzuhti, si deux oranges sont entassées de façon ordonnée, ou de churu si les oranges sont entassées de façon désordonnée. Le locuteur peut donc effectuer un choix qui est lié à sa propre représentation de la situation.

La présentation ci-dessus en groupes de deux ou trois indices permet de mettre en valeur les traits sémantiques des indices mais pas la dynamique de ces unités. Dans son étude sur les classificateurs du purépecha préhispanique, Fernando Nava effectue une présentation de ces unités sous une forme hiérarchisée. Le centre est représenté par les trois unités qui existent encore aujourd'hui. Ses hypothèses concernant l'organisation des unités recoupent certaines présentées ci-dessous et divergent en plusieurs points. Il regroupe seize unités (il écarte quira et chungua par manque d'informations) en sept groupes :

(1) irha - ycha - echu

[rond] - [long] - [plat]

(2) para - anga - çutu

[rond $] /[$ singulier $]$ - [long $] /[$ singulier $]$ - [plat $] /[$ singulier $]$

(3) thsinguui - hutu - tacu

[rond]/[pluriel]- [long]/[pluriel] - [plat]/[pluriel]

(4) xancha - chuui

$[+$ proéminent $] /[+$ long $]$ - $[$-proéminent $] /[+$ rond $] /[+$ plat $]$

3 (5) thumbi - chere

[+volumineux $]$ - [-volumineux]

(6) umba - churu

[+animé]/[pluriel] - [-animé]/[pluriel]

(6') umba - tzuhti

[+animé]/[pluriel] - [-animé]/[duel]

\footnotetext{
4 L'opposition entre le pluriel et le duel, présente pour les objets entassés de façon ordonnée, est assez originale pour cette langue.
} 
On peut questionner en particulier l'utilisation du nombre (singulier et pluriel) pour établir deux groupes d'unités. Dans le groupe des unités qui apparaissent avec des objets au "singulier", on trouve çutu et para. On peut s'interroger sur l'emploi exclusif de ces éléments pour le dénombrement d'un seul objet. De même, l'utilisation du critère [animé] ne semble pas correspondre à la description faite par les auteurs. Ils expliquent que les deux unités umba et churu accompagnent des choses telles des oranges. L'unité umba peut apparaître avec des termes désignant des personnes mais ce n'est pas une exclusité. Il ne semble donc pas se dessiner une opposition.

Il me semble donc essentiel pour analyser les informations fournies par Gilberti et Lagunas et émettre des hypothèses qui servent de base pour comprendre l'évolution des indices catégoriels en purépecha d'aller au-delà de ces présentations (celle de Fernando Nava ou celle qui se trouve ci-dessus) et de montrer comment ces unités s'organisent au sein d'un continuum :

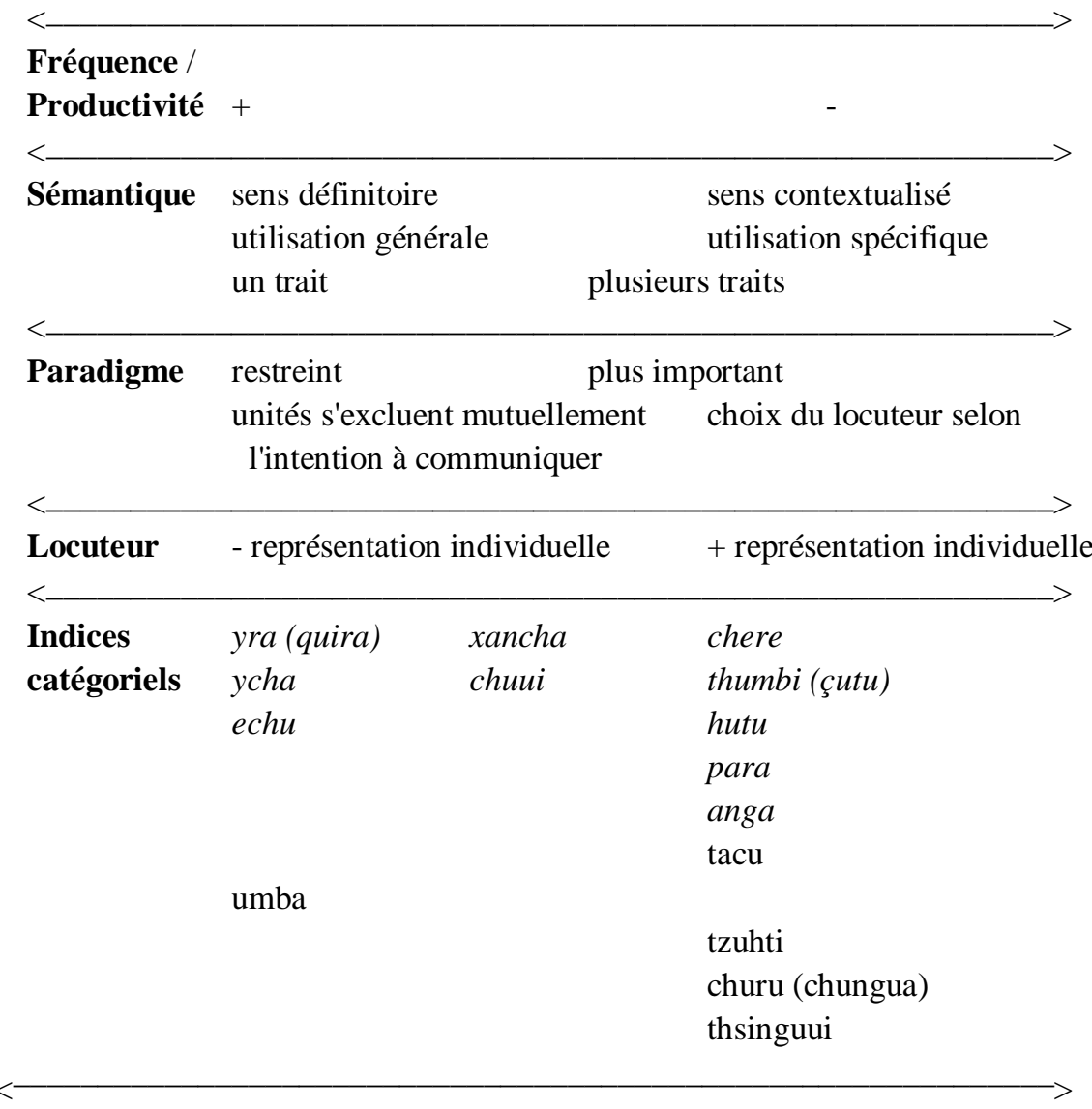

Cette représentation permet d'appréhender l'hétérogénéité et les tendances dynamiques de l'ensemble des indices catégoriels existants au XVIème siècle. On peut remarquer que les unités les plus productives sont celles dont l'apparition n'est conditionnée que par un trait sémantique de l'objet (en référence à sa description physique). Les unités les moins productives requièrent une analyse plus précise de la situation de la part du locuteur 5 .

\section{LES PROCESSUS DE SIMPLIFICATION PENDANT LA PERIODE COLONIALE}

4

Entre le XVII ème et le XIXème siècle, il y eut peu de descriptions du purépecha. Deux d'entre elles présentent les indices catégoriels.

En 1714, dans son Arte de la lengua tarasca, Diego Basalenque reprend l'inventaire effectué par Maturino Gilberti. Cependant, il innove en rangeant les unités par ordre alphabétique6. Cette présentation neutre et arbitraire

5 Ces hypothèses reposent sur l'analyse des données fournies par Gilberti et Lagunas. Cependant, seul le sens qui est fourni par les auteurs peut être utilisé et ils présentent très peu d'exemples. De plus, certains éléments sont désignés comme des synonymes, le sont-ils vraiment ou existe-t-il des différences significatives qui pourraient se révéler pertinentes pour l'analyse ? Les présentations de Gilberti et Lagunas, bien que remarquables, ne nous permettent pas de savoir si ces unités étaient utilisées obligatoirement dans tous les contextes et si elles étaient employées avec tous les noms.

6 Basalenque, 1962 [1714], p. 147. 
correspond à la disposition choisie par Basalenque pour présenter les prépositions, les suffixes, etc... Ce choix apporte davantage de clarté à son étude par rapport aux œuvres de ses aînés. Mais, aucune information nouvelle n'apparaît par rapport à l'étude de Gilberti en ce qui concerne les indices catégoriels?

Dans l'inventaire qu'effectue Najera 8 , en 1831, il manque six unités par rapport à la présentation de Gilberti (yra, quira, ycha, chere, tzuhti, chungua). Cette simplification témoigne de l'évolution de la langue mais peut aussi être la conséquence d'une présentation tronquée. En effet, deux indices catégoriels qui sont utilisés aujourd'hui ( $y r a$ et ycha) et dont on peut penser qu'ils existaient aussi au XIX ${ }^{e ̀ m e}$ siècle ne sont pas présents. Les indices présentés par Najera, par ordre alphabétique, sont les suivants :

\begin{tabular}{ll}
\hline Indices catégoriels & Sens \\
\hline anga & en hauteur \\
churu (umba) & entassé sans ordre \\
chuui (xancha) & sur pied \\
echu (tacu) & plat \\
hutu & en bottes \\
para & séparés \\
thsinguui & entassé (l'un dans l'autre) \\
thumbi $($ çutu) & sur le sol \\
\hline
\end{tabular}

Pour Najera, il existe trois couples de variantes, en plus de celles présents chez Gilberti : echu-tacu, churuumba, chuui-xancha. Cette nouveauté peut être révélatrice de l'évolution du sens des indices. En effet, tacu et echu renvoient tous les deux uniquement à la caractéristique [plat], le premier terme ne renvoie plus à l'agencement des objets. Ce qui distinguait umba et churu était le type d'ordonnance des objets entassés. Cette opposition est neutralisée, seul le trait [-ordonné] persiste. Les unités xancha et chuui se différenciaient par le nombre d'éléments de soutien des objets. La distinction n'existe plus. L'unité para indique seulement que les objets sont séparés. De même, thsinguui s'utilise pour marquer l'entassement ordonné de choses. Le sens de thumbi (ou çutu) s'est déplacé pour désigner des objets qui se localisent sur le sol et anga désigne des objets qui se situent en hauteur. La présentation de Najera est de nature à apporter des informations concernant les processus de simplification quantitative et sémantique mais aussi sur le déplacement du sens de certains indices catégoriels.

\section{LA REDUCTION ET LA REORGANISATION DES INDICES CATEGORIELS AU XXEME SIECLE}

Mary Foster présente dix indices catégoriels ${ }^{9}$. Cependant, seuls trois éléments ( $i \ll a$, itSa et itSu) ${ }^{10}$ permettent de catégoriser les unités de la classe des noms. Ces trois unités s'utilisent avec le suffixe - $k u a$ qui permet, en particulier, de former des noms. Elle définit ainsi ces trois unités :

- i rakua : "objet rond" (énumérations d'objets ronds incluant les femmes et les filles)

- it fakua : "objet long, rigide" (énumérations d'objets longs dont les garçons et les chiens)

- it fukua : "objet plat, fin" (énumérations d'objets plats comme les feuilles, les tortillas)

Elle illustre cette présentation avec différents énoncés, par exemple (p. 159) :

5

\footnotetext{
7 Je ne pense pas, comme le dit Fernando Nava, que par ce choix de présentation, Basalenque a voulu montrer que certains éléments présentent des signes d'improductivité. Nava, 1997, p. 307.

8 Najera, 1944 [1831], p. 42-43.

9 Foster, 1969, p. 158.

10 L'écriture adoptée ici est l'alphabet phonétique internationale. Le terme itSu représente une variante dialectale de $e$ t.Su (echu).
} 
1. tanimu-erakua uari -itfa

trois -[rond] 11 femme-pl.

Trois femmes

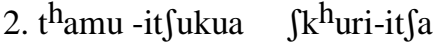 \\ Quatre-[plat] feuille-pl. \\ Quatre feuilles.}

Les sept autres éléments 12 présentés comme des indices catégoriels n'accompagnent pas obligatoirement un nom et fonctionnent différemment, par exemple (p. 159) :
3. $\mathrm{u} \quad-\mathrm{a} \quad-\mathrm{ka}=$ ni
tani -nkuiripu -nkuni
Faire-fut-ass $1 / 2=1$
trois-[individu]+com
Je ferai avec trois personnes.

Dans l'exemple 3, kuiripu signifie "individu" ou "personne" et fonctionne comme un nom et pas comme un indice catégoriel qui accompagne un nom13. L'unité kuiripu peut apparaître sans numéral : u-a-ka-ni kuiripu-nkuni "Je ferai avec la personne."

En 1970, Paul Friedrich ${ }^{14}$ présente de façon détaillée les trois indices catégoriels utilisés en purépecha (i $r a$, it $\int a$ et $i t \int u$ ). Il indique qu'ils ont une grande fréquence et qu'ils s'excluent mutuellement. Ils accompagnent certains noms et caractérisent la forme et la dimension de l'objet référé par le nom. Ne sont pas accompagnés d'un indice, les noms ayant pour référent une masse, un liquide, un humain et les noms abstraits. Sous certaines conditions, les noms qui désignent des humains peuvent apparaître avec des indices catégoriels.

Friedrich définit ainsi les trois unités :

- it $\int a$ : long et unidimensionnel (il accompagne par exemple les noms d'arbres)

-itfu : plat et bidimensionnel (il accompagne par exemple : tortilla, cuir, tissu)

- ira : rond et tridimensionnel (il accompagne par exemple : maison, pot, balle)

Friedrich insiste sur le fait que le choix des indices catégoriels dépend de la perception des formes et des dimensions des objets par l'ensemble de la communauté, il s'agit donc d'un fait culturel. Il expose quelques cas dans lesquels la situation peut influencer l'utilisation d'un indice plutôt qu'un autre. Un ballon de baudruche non gonflé sera accompagné de it fa, lorsqu'il est gonflé c'est $i \ll a$ qui sera présent. Les noms ayant pour référents des humains ne sont pas accompagnés d'un indice catégoriel sauf dans certaines situations où l'on veut faire ressortir des caractéristiques physiques. Ainsi, par exemple, le nom ua ziti "femme" pourra être accompagné de $i \ll a$, afin d'insister sur la particularité de la femme désignée (la rondeur). Lorsqu'un objet peut être caractérisé par deux formes, c'est la perception et l'interprétation personnelles du locuteur qui transparaîtront (par exemple, le ruban peut être accompagné de itfa ou de $i t S u$ ). Finalement, Friedrich effectue une remarque très intéressante en précisant que l'indice catégoriel peut avoir un rôle pertinent dans le cas d'homonymie. Par exemple, le nom karast signifie "ver de terre" et "pomme sauvage". Pour distinguer les deux sens, il peut être accompagné dans le premier cas de it fa et dans le deuxième cas de $i \ll a$. Cette analyse détaillée prouve la productivité et la vitalité des trois indices catégoriels.

11 Les abréviations utilisées sont : aor. : aoriste, ass. : assertif, com. : comitatif, dém. : démonstratif, fut. : futur, hab. : habituel, obj. : objet, pl. : pluriel, entre crochets [ ] est indiqué le sens de l'indice catégoriel.

12 Ces unités sont : $n$ kuiri pu "personne" (Il est utilisé dans les énumérations de personnes incluant les hommes, les femmes, les filles mais pas les garçons.), nta "groupe de", nta mpe ri "temps", nu ri a "période de temps, intervalle", pera "dans la partie de", pu«u "groupe de", «u $t S a$ "...par...".

13 Il n'est pas aisé d'expliquer la présence du $n$ qui est antéposé à kuiripu. Il ne peut s'agir d'un préfixe au nom kuiripu, puisque le purépecha se particularise par l'utilisation exclusive de suffixes. Il s'agit donc d'une unité suffixée au nombre :
$\begin{array}{llll}\mathrm{u} & -\mathrm{a} & -\mathrm{ka} & -\mathrm{ni}\end{array}$
tani-ni?
kuiripu -nkuni
faire+fut.+ass. $1 / 2+1 \quad$ trois + personne+com.

On peut penser aussi que les différents éléments forment un composé tani-n-kuiripu. L'unité $n$ serait alors un élément d'appui tel qu'il apparaît par exemple dans certaines réduplications : tsuntsu "marmite"

tSentSeki "âne"

14 Friedrich, 1970, p. 381-386. 


\section{A L'AUBE DU XXIEME SIECLE, EXISTE-T-IL ENCORE DES INDICES CATEGORIELS EN PUREPECHA ?}

Dans le corpus que j'ai recueilli15, trois indices catégoriels apparaissent. Ces unités se présentent toujours avec le suffixe -kua. Les indices sont toujours postposés à un numéral.

- itfakua : apparait neuf fois dans le corpus et accompagne les noms suivants : "corps, doigt, main, enfant, arbre, chien, poulet et tortilla".

- i qakua : apparaît une fois et accompagne le nom "pomme".

- it fukua : apparaît deux fois et accompagne le nom "tortilla".

Au niveau de la valeur, on peut constater que itfakua accompagne, le plus souvent, les noms d'objets qui ont une forme longue : corps, doigt, main, enfant, et arbre. En revanche, trois objets ne sont pas caractérisés par la longueur : poulet, chien et tortilla. L'élément i rakua accompagne le nom d'un objet qui a une forme ronde et it fukua apparaît avec le nom d'un objet de forme plate "tortilla". Certains informateurs indiquent que le nom "tortilla" ne peut être accompagné que de itfukua mais pour d'autres locuteurs de cette langue, on peut utiliser soit itfukua soit it fakua pour accompagner "tortilla".

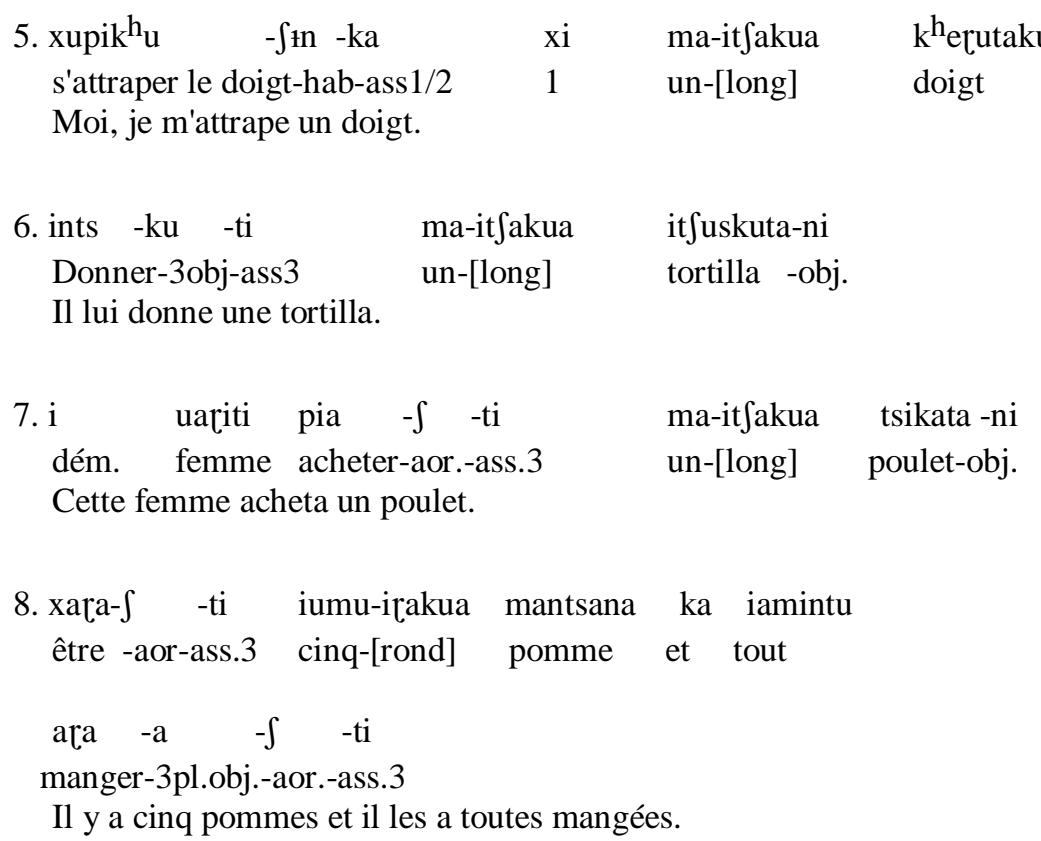

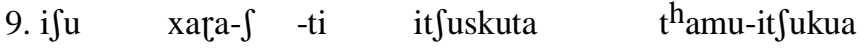
ici être-aor-ass.3 tortilla quatre-[plat]
Ici, il y a quatre petites tortillas.

Dans l'exemple 9, on peut remarquer que le numéral et l'indice catégoriel sont postposés au nom. Généralement, ils

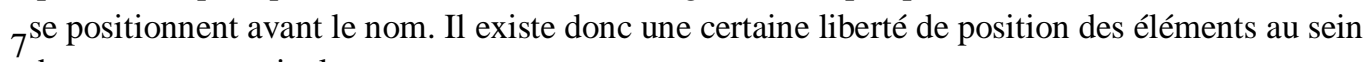
du syntagme nominal.

Le nombre d'exemples recueillis est peu important, il témoigne donc de la rareté de l'utilisation de ces éléments. Ces exemples permettent néanmoins d'effectuer certaines remarques sur la dynamique actuelle de ces trois unités :

- L'utilisation des indices est rare. Seuls douze exemples sont présents dans le corpus. L'unité itfakua est plus fréquente que les deux autres, ses occurrences représentent $75 \%$ des exemples.

15 Le corpus a été recueilli à Jaracuaro, presquîle du lac de Patzcuaro (Michoacan). 
- La réduction du nombre des unités au sein de la langue purépecha entraîne une économie paradigmatique. Elle a pour conséquence une simplification dans laquelle sont conservés les indices qui étaient les plus fréquents et les plus productifs au XVIème siècle. Il s'agit des éléments dont le sens est lié à la définition même des objets, à leur propriété générale. Ils sont, par conséquent, d'une utilisation plus aisée et se présentent comme plus propices à assumer le rôle d'indice catégoriel.

- Au niveau sémantique, des changements s'effectuent. Le passage progressif d'un système de dix-huit éléments à l'existence de trois unités entraîne un déplacement du sens des indices catégoriels. L'unité itfakua accompagne des noms tels "chien", "poulet" et "tortilla" qui n'ont pas pour caractéristique physique la longueur 16 . Cette réorganisation s'accompagne d'une démotivation du sens des indices catégoriels. L'utilisation fluctuante de itfukua ou itfakua avec le nom "tortilla" (exemples 6 et 9) prouve que certains locuteurs hésitent sur le sens des unités. Pour ces locuteurs, il existe dans ce contexte, une équivalence sémantique entre it fukua et itfakua. L'unité it fakua peut être employée avec un nom se référant à un objet plat. En revanche, ils refusent l'utilisation de itfukua avec un nom qui se réfère à un objet long. Autrement dit, l'équivalence sémantique n'est possible qu'avec des noms qui font référence à des objets plats. La dynamique actuelle marque non seulement une plus grande fréquence de itfakua mais aussi une extension de son sens :

- itfakua : long / plat

- itfukua : plat

- irakua : rond

- La présence de ces éléments est libre et arbitraire. Elle n'est fondée sur aucune explication possible et ne répond pas à une volonté de catégoriser systématiquement les unités de la classe des noms. Elle n'est pas contrainte par la présence d'un numéral. En revanche, un indice catégoriel est toujours accompagné d'un numéral. Les indices catégoriels n'apparaissent qu'avec neuf unités de la classe des noms. Par exemple, ils accompagnent 6\% des occurrences du nom "tortilla" et $1 \%$ des occurrences du nom "chien" présentes dans le corpus (dans un contexte de quantification). Leur utilisation renvoie peut-être à un procédé stylistique qui permet de focaliser l'objet référé. Ils ne permettent plus d'établir des catégories au sein de la classe des noms et ne peuvent pas être considérés aujourd'hui comme des indices catégoriels mais plutôt comme les vestiges d'un ancien système d'indices catégoriels.

Cette analyse met en lumière l'utilisation des indices catégoriels aujourd'hui à Jaracuaro. La variante de la langue purépecha parlée dans ce village (et dans cette région) est particulièrement menacée de disparition. La rareté d'utilisation des indices catégoriels est peut-être dialectale, il est possible que dans d'autres variantes, ces éléments soient utilisés plus fréquemment et de façon moins fluctuante. Une étude permettant la comparaison des différents dialectes de cette langue fournirait probablement une réponse.

\section{BIBLIOGRAPHIE}

BASALENQUE Diego, 1962 [1714], Arte de la lengua tarasca, Morelia, Editorial Erandi, 163 p.

FOSTER Mary, 1969, The tarascan Language, Berkeley, University of California Press, 200 p.

FRIEDRICH Paul, 1970, Shape in Grammar, in Language, 46, 2, p. 379-407.

${ }^{8}$ GILBERTI Maturino, 1987 [1558], Arte de la lengua de Michuacán, Morelia, Fimax, XCII + 344 p. (Introduction de J. Benedict Warren)

LAGUNAS Juan Bautista de, Arte y dictionario con otras obras en lengua de Michuacana, Morelia, Fimax,XXIII + 519 p. (Introduction de J. Benedict Warren)

NAJERA de San Juan Crisostomo Manuel, 1944 [1831], Gramática de la lengua tarasca, Mexico, Libros de Mexico, $72 \mathrm{p}$.

16 Dans son étude, Friedrich indique que les noms d'animaux sont généralement accompagnés de itSakua. 
NAVA Fernando, 1997, Los clasificadores numerales del p'urhépecha prehispánico, in Anales de Antropología, 31, p. 299-309. 